

\title{
Effect of natriuretic peptide-guided therapy on hospitalization or cardiovascular mortality in high-risk patients with heart failure and reduced ejection fraction: a randomized clinical trial
}

\author{
G. Michael Felker, MD, MHS ${ }^{\star}$, Kevin J. Anstrom, PhD, Kirkwood F. Adams, MD ${ }^{\dagger}$, Justin A. \\ Ezekowitz, MBBCh, MSc ${ }^{\ddagger}$, Mona Fiuzat, PhD*, Nancy Houston-Miller, RN, BSN ${ }^{\star}$, James L. \\ Januzzi Jr., MD", Daniel B. Mark, MD, MPH${ }^{\star}$, Ileana L. Piña, MD, MPH , Gayle Passmore, \\ PMP $^{\star}$, David J. Whellan, MD, MHS\#, Hongqiu Yang, PhD*, Lawton S. Cooper, MD, MPH ${ }^{\star \star}$, \\ Eric S. Leifer, PhD ${ }^{* *}$, Patrice Desvigne-Nickens, MD ${ }^{* *}$, and Christopher M. O’Connor, MD ${ }^{\star}, \dagger \dagger$ \\ *Duke Clinical Research Institute, Durham, NC, USA \\ †University of North Carolina-Chapel Hill, Chapel Hill, NC, USA \\ ¥Canadian VIGOUR Centre, University of Alberta, Edmonton, Canada \\ $\S$ The LifeCare Company, Menlo Park, CA, USA \\ "Cardiology Division, Massachusetts General Hospital, Boston, MA, USA \\ "Albert Einstein College of Medicine, Bronx, NY, USA \\ \#Thomas Jefferson University, Philadelphia, PA, USA \\ ${ }^{* *}$ Division of Cardiovascular Sciences, National Heart Lung and Blood Institute, Bethesda, MD, \\ USA \\ t+Inova Heart and Vascular Center, Fairfax, VA, USA
}

\begin{abstract}
IMPORTANCE-The natriuretic peptides are biochemical markers of heart failure (HF) severity and predictors of adverse outcomes. Smaller studies have evaluated adjusting HF therapy based on natriuretic peptide levels ("guided therapy") with inconsistent results.

OBJECTIVE-To determine whether an amino-terminal pro-B-type natriuretic peptide (NTproBNP)-guided treatment strategy improves clinical outcomes compared to usual care in highrisk patients with $\mathrm{HF}$ and reduced ejection fraction (HFrEF).
\end{abstract}

DESIGN, SETTINGS, AND PARTICIPANTS-The GUIDing Evidence Based Therapy Using Biomarker Intensified Treatment in Heart Failure (GUIDE-IT) study was a randomized multicenter clinical trial conducted between January 16, 2013 and September 20, 2016 at 45 clinical sites in the United States and Canada. This study planned to randomize 1,100 patients with HFrEF (ejection fraction $\$ 40 \%$ ), elevated natriuretic peptide levels within the prior 30 days, and a history of a prior HF event (HF hospitalization or equivalent) to either an NT-proBNP-guided strategy or usual care.

INTERVENTION-Patients were randomized to either an NT-proBNP-guided strategy or usual care. Patients randomized to the guided strategy $(n=446)$ had HF therapy titrated with the goal of achieving a target NT-proBNP $<1,000 \mathrm{pg} / \mathrm{mL}$. Patients randomized to usual care $(\mathrm{n}=448)$ had 
heart failure care in accordance with published guidelines, with emphasis on titration of proven neurohormonal therapies for heart failure. Serial measurement of NT-proBNP testing was discouraged in the usual care group.

MAIN OUTCOMES AND MEASURES-The primary endpoint was the composite of time-tofirst HF hospitalization or cardiovascular mortality. Prespecified secondary endpoints included allcause mortality, total hospitalizations for HF, days alive and not hospitalized for cardiovascular reasons, the individual components on the primary endpoint, and adverse events.

RESULTS-The Data and Safety Monitoring Board recommended stopping the study for futility when 894 (median age, 63; 286 (32\%) women) of the planned 1,100 patients had been enrolled and followed for a median of 15 months. The primary endpoint occurred in 164 patients (37\%) in the biomarker-guided group and 164 patients (37\%) in the usual care group (adjusted hazard ratio $=0.98 ; 95 \%$ confidence interval $0.79-1.22 ; \mathrm{p}=0.88$ ). Cardiovascular mortality was $12 \%$ in the biomarker guided group and $13 \%$ in the usual care group (hazard ratio $=0.94$ (95\% confidence interval $0.65-1.37, \mathrm{p}=0.75$ ). Neither other secondary endpoints nor achieved decreases in NTproBNP levels were significantly different between the groups.

CONCLUSIONS AND RELEVANCE-In high-risk patients with HFrEF, a strategy of NTproBNP-guided therapy was not more effective than a usual care strategy in improving outcomes.

TRIAL REGISTRATION—Clinicaltrials.gov identifier: NCT01685840

\section{Keywords}

amino-terminal pro-B-type natriuretic peptide; heart failure with reduced ejection fraction; patient outcomes

Evidence-based therapies such as targeting neurohormonal activation significantly improve outcomes in patients with heart failure (HF). Nevertheless, available data suggest that many patients in clinical practice are either not treated with these agents or are treated with lower than recommended doses. ${ }^{1,2}$ The natriuretic peptides, specifically B-type natriuretic peptide (BNP) and amino-terminal pro-B-type natriuretic peptide (NT-proBNP), are biomarkers that reflect HF severity and are significantly associated with adverse outcomes in HF. ${ }^{3,4}$ These markers decline in response to the use of guideline-recommended HF therapies, and rising levels portend a poor prognosis. ${ }^{5}$ These observational data have led to the hypothesis that serial measurements of natriuretic peptides may be used to guide titration of chronic medical therapy in HF.

Previous clinical trials of varying size and design have tested this hypothesis over the last two decades, with mixed results. ${ }^{6-11}$ These studies have generally been limited by their small size and also by significant heterogeneity between studies. Several meta-analyses have suggested substantial benefits with this approach, but no individual study has been of sufficient power to be definitive. ${ }^{12,13}$ In light of this uncertainly, current guidelines do not recommend the use of serial measurements of natriuretic peptides to guide titration of HF therapy. ${ }^{14,15}$ The Guiding Evidence Based Therapy Using Biomarker Intensified Treatment in Heart Failure (GUIDE-IT) multicenter randomized clinical trial was designed to evaluate 
the efficacy of a NT-proBNP-guided HF treatment strategy compared to optimal medical therapy alone in high-risk patients with heart failure and reduced ejection fraction (HFrEF).

\section{Methods}

\section{Study Design}

The details of the rationale and design for this study have been published previously. ${ }^{16}$ The study protocol, including the statistical analysis plan, is provided in the Supplemental Material. The study was approved by the institutional review board at each study site, and all participants provided written informed consent. An independent Data and Safety Monitoring Board (DSMB) appointed by the National Heart Lung and Blood Institute monitored study conduct and patient safety. In order to maximize adherence to the study protocol, an adherence committee reviewed episodes in which HF therapy was not titrated despite NTproBNP values being above the target and provided general feedback to the Executive Committee and the study sites (including study site score cards indicating cumulative site performance with regard to protocol adherence) on a regular basis. The adherence committee had a stepped approached for sites with consistently poor performance, including contact from the coordinating center and escalation to the Executive Committee to reinforce study goals and site training.

\section{Study Participants}

Patients were eligible for enrollment if they had chronic HFrEF with an ejection fraction of $40 \%$ or less, a history of a prior HF event (hospitalization for HF, emergency department visit for $\mathrm{HF}$, or outpatient treatment with intravenous diuretics for $\mathrm{HF}$ ) within the prior 12 months, and an NT-proBNP $>2,000 \mathrm{pg} / \mathrm{mL}$ or BNP $>400 \mathrm{pg} / \mathrm{mL}$ within the prior 30 days. Patients were excluded if they had an acute coronary syndrome or revascularization procedure within the prior 30 days, cardiac resynchronization therapy within the prior 3 months, end-stage renal disease, or anticipated heart transplantation or mechanical cardiac support within the next 12 months. In accordance with NIH policy, patient reported race and ethnicity information was collected using fixed categories.

\section{Randomization and Treatment Assignments}

Enrolled patients were randomized in a 1:1 fashion using computer generated random numbers using a simple randomization design with no restrictions to either the NT-proBNPguided therapy strategy or usual care. Given the nature of the study intervention, treatment assignment was not blinded. For patients randomized to the NT-proBNP-guided strategy, clinicians were instructed to titrate HF therapy to target an NT-proBNP level $<1,000 \mathrm{pg} / \mathrm{mL}$. Specific adjustments of therapy for individual patients were at the discretion of the treating physician, but sites were encouraged to prioritize titration of neurohormonal antagonists over diuretics unless there was clinical evidence of congestion or volume overload. Patients randomized to the NT-proBNP-guided group used local laboratory NT-proBNP measurements to make decisions about titration of HF therapy. All patients in either group also had blinded NT-proBNP concentrations measured in a core laboratory at each study visit. For patients in either group, investigators were provided with the most recent American Heart Association (AHA)/American College of Cardiology (ACC) practice 
guidelines for the management of HF and specific information on target doses of proven medical therapies. After an initial visit at 2 and 6 weeks, visits occurred every 3 months throughout the remainder of the study. After therapy adjustment for HF (whether driven by NT-proBNP levels or clinical reasons), patients had a 2-week follow-up visit for reassessment.

\section{Study Outcomes}

The primary outcome was a composite of time-to-first HF hospitalization or death from cardiovascular causes. Prespecified secondary endpoints included all-cause mortality, total hospitalizations for HF, days alive and not hospitalized for cardiovascular reasons, the individual components on the primary endpoint, health related quality of life, resource utilization, costs, cost effectiveness, and safety. Results of the economic and quality of life analyses are not reported in this article. Adjudication of all deaths and hospitalizations was carried out by a blinded clinical endpoint committee according to pre-specified criteria. We predefined four adverse events of interest that might be anticipated to occur more frequently with more aggressive HF treatment: symptomatic hypotension, symptomatic bradycardia, hyperkalemia, and worsening renal function.

\section{Statistical Analysis}

A total sample size of 1,100 patients (550 per group) was expected to provide approximately $90 \%$ power to detect a difference in the primary endpoint with an assumed type I error rate of 0.05 two-sided. We estimated that the annual event rate for the composite endpoint would be $40 \%$ in the usual care group. We targeted a $20 \%$ decrease in the primary endpoint at 12 months for the biomarker-guided group in the sample size calculation, based on the recognition that this treatment effect would be consistent with other effective heart failure therapies that have been incorporated into clinical practice. ${ }^{17}$ According to protocol, all patients were to be followed for between 12 and 24 months after randomization (the last patient enrolled to be followed for 12 months). For the analysis of the primary endpoint, the adjusted hazard ratio would be adjusted for 5 prespecified baseline covariates-age, sex, ejection fraction, NT-proBNP level, and the presence of diabetes mellitus-within the Cox regression model. For missing baseline categorical variables, we imputed the most common value. For missing baseline NT-proBNP values, we utilized the NT-proBNP value from screening. For missing baseline ejection fraction values, we imputed the population median. We also performed the primary endpoint analysis with site as a random effect as a sensitivity analysis. We tested for heterogeneity of effect on the primary endpoint by testing for interactions within a number of subgroups defined by demographics and baseline characteristics (see Online Supplement). A subgroup analysis based on age ( 275 years vs. < 75 years) was prespecified based on prior data suggesting biomarker guided therapy was more effective in younger patients. ${ }^{9}$ For secondary analyses, inverse probability weighting was used to estimate mean days alive out of the hospital using the Bang-Tsiatis partitioned estimator. ${ }^{18}$ The total number of recurrent HF hospitalizations by treatment group was modelled using the Andersen-Gill intensity model. ${ }^{19}$ All analyses were based on the principle of intention to treat. All analyses were performed using SAS 9.4. The threshold for statistical significance was 2 sided with a Type 1 error rate of 0.05 . There was no adjustment 
performed for multiple comparisons and thus secondary outcomes were considered exploratory.

\section{Results}

\section{Study Patients}

A total of 894 patients were enrolled at 45 sites in the United States and Canada between January 2013 and July 2016 (Figure 1). The groups were generally well balanced with respect to baseline characteristics (Table 1). The study enrolled patients with high-risk HF, as characterized by a low ejection fraction (median 25\%), significantly elevated NT-proBNP (median 2,653 pg/mL), and a history of prior HF hospitalization (or equivalent) in the past year. Most patients were receiving recommended pharmacologic therapy for chronic heart failure at baseline. The median-follow up time for all patients was 15 months. Missing data for the 5 prespecified adjustment covariates was rare (none for age or sex, 1 for diabetes mellitus, 14 for baseline NT-proBNP, and 12 for ejection fraction).

At the regularly scheduled DSMB meeting on July 8, 2016, at which time about $50 \%$ of planned primary endpoint events had occurred, the study met prespecified inefficacy criteria and the DSMB made a recommendation to the NHLBI to discontinue the study due to lack of efficacy evidence for the biomarker-guided treatment group compared to usual care. The NHLBI accepted this recommendation and enrollment was discontinued after 894 patients had been enrolled ( $81 \%$ of planned enrollment). Final study visits for all patients still actively participating in the trial were completed prior to database lock.

\section{Medical Treatment by Strategy and Follow-up}

Patients randomized to the biomarker-guided strategy had a greater number of study clinic visits (median 12 vs. 10, Wilcoxon $\mathrm{p}=0.002$ ) and more adjustments to HF therapy (median 6 vs. 4, Wilcoxon $\mathrm{p}<0.001$ ) compared to patients randomized to usual care. Over the course of the study, there was modest intensification of HF therapy in both groups, without statistically significant differences between those randomized to NT-proBNP-guided therapy or usual care (Table 2).

\section{Study Outcomes}

The composite endpoint of first hospitalization for HF or death from a cardiovascular cause occurred in 164 patients (37\%) in the biomarker-guided group and 164 patients (37\%) in the usual care group with 12-month Kaplan-Meier event rates of 33.8\% and 36.0\%, respectively and a treatment difference of $-2.2 \%$ (95\% confidence interval $[\mathrm{CI}]-9.1 \%$ to $4.6 \%$ ). After adjustment for prespecified covariates, the adjusted hazard ratio for the primary endpoint was 0.98 (95\% CI $0.79-1.22, \mathrm{p}=0.88$ ) (Figure 2 ). These results were not significantly changed by including site as a random effect $(\mathrm{HR}=0.99,95 \% \mathrm{CI} 0.79-1.23, \mathrm{p}=0.92)$. Data for all-cause mortality, cardiovascular mortality, HF hospitalization, and all-cause hospitalization were also not significantly different between treatment groups (Table 3). Death occurred in 66 patients (15\%) in the biomarker-guided group and $77(17 \%)$ in the usual care group. The 12-month Kaplan-Meier event rates for all-cause mortality were $9.8 \%$ for biomarker-guided and $14.1 \%$ in the usual-care group for a treatment difference of $-4.3 \%$ 
( $95 \%$ CI $-8.9 \%$ to $0.3 \%$ ). After adjustment for the pre-specified covariates, the adjusted hazard ratio for all-cause mortality was $0.86(95 \%$ CI $0.62-1.20$; $\mathrm{p}=0.37)$.

There was generally no evidence of heterogeneity of treatment effect in a number of prespecified and post-hoc subgroups (eFigure 1). Changes in the concentrations of NTproBNP (based on blinded central core lab data) decreased over time in both groups and were not significantly different between groups; at 12 months, median NT-proBNP had decreased from a median of 2,568 to $1,209 \mathrm{pg} / \mathrm{mL}$ (53\% decrease) in the biomarker-guided group, and from a median of 2,678 to $1,397 \mathrm{pg} / \mathrm{mL}$ (48\% decrease) in the usual care group (Figure 3). The proportion of patients in both groups achieving the target value of NTproBNP $<1,000 \mathrm{pg} / \mathrm{mL}$ at 12 months was $46 \%$ for biomarker-guided group vs. $40 \%$ for usual care group $(\mathrm{p}=0.21)$.

\section{Adverse Events}

The rates of the predefined adverse events of interest (i.e., symptomatic hypotension, symptomatic bradycardia, hyperkalemia, and worsening renal function) were generally low and similar between the groups (eTable 1).

\section{Discussion}

The primary finding of this study is that in high-risk patients with HFrEF, a strategy of guiding therapy based on concentrations of NT-proBNP was not more effective than a usual care strategy in reducing the composite endpoint of time-to-first HF hospitalization or cardiovascular death. Similarly not significantly different results were seen in other clinical endpoints. Although there were more adjustments to therapy in the biomarker-guided group, neither doses of guideline-directed medical therapy, the achieved NT-proBNP concentrations, nor clinical outcomes were significantly different between the treatment groups.

These results differ from other data, including a recent comprehensive patient-level metaanalysis of data from 2,431 patients from 11 trials which showed a reduction in all-cause mortality with natriuretic peptide-guided therapy compared to usual care (hazard ratio = 0.62). ${ }^{13}$ A consistent feature of other studies in which natriuretic peptide-guided therapy was shown to be effective was the differential utilization of neurohormonal therapies as well as a separation of achieved natriuretic peptide concentrations between the two study groups. The up-titration of medical therapy in the NT-proBNP group in this study was substantially less than that seen in some smaller studies of biomarker-guided therapy. For example, a randomized study of 278 patients in eight Austrian hospitals achieved 100\% of angiotensinconverting enzyme (ACE) inhibitor or angiotensin receptor blocker (ARB) target doses and $77 \%$ of beta-blocker target doses in patients randomized to biomarker-guided therapy, which was accompanied by a substantial reduction in HF events. ${ }^{10}$ Although it is challenging to compare across studies, the achieved dosing of these classes of drugs in the NT-proBNPguided group was substantially less in this study ( $55 \%$ for ACE/ARB and $48 \%$ for betablockers at 12 months, Table 2). Whether the lack of up-titration of medical therapy observed in this study was related to patient characteristics (e.g., inability to up-titrate due to azotemia or hypotension) or physician behavior (e.g., unwillingness to up-titrate due to 
concern over adverse effects) in not clear from these data. This study enrolled patients with high-risk features (elevated natriuretic peptide levels within the prior 30 days and an HF event within the prior 12 months) and allowed a broad range of renal function, resulting in a study population with relatively advanced HF compared to most other clinical trials in ambulatory patients with HFrEF. By way of comparison, the median baseline NT-proBNP value in this study $(2,607 \mathrm{pg} / \mathrm{mL})$ was 1.6 fold that of patients enrolled in the Prospective Comparison of ARNI with ACEI to Determine Impact on Global Mortality and Morbidity in Heart Failure (PARADIGM-HF) study of sacubitril/valsartan ${ }^{17}$ and 3.2 fold that of patients enrolled in the Heart Failure and a Controlled Trial Investigating Outcomes of Exercise Training (HF-ACTION) study. ${ }^{20}$ Patients with more severe HF such as those in this study may have more limitations to intensification of HF therapy, in particular hypotension and azotemia, which may have limited this ability to aggressively up-titrate medical therapy in the guided therapy group in response to above target NT-proBNP levels.

Another potential difference between this study and other data may relate to difference in the control group. In the single center ProBNP Outpatient Tailored Chronic HF Therapy (PROTECT) study, patients randomized to biomarker-guided therapy achieved a $44 \%$ decrease in the NT-proBNP level over time (compared to a 5\% decrease in the usual care group), which was associated with a significant improvement in clinical outcomes for those patients randomized to the NT-proBNP-guided strategy. ${ }^{8}$ By contrast, in the current trial, both the decrease in NT-proBNP concentrations (Figure 3) and the proportion of patients in each group who reached the target NT-proBNP value of $<1,000 \mathrm{pg} / \mathrm{mL}$ ( $46 \%$ vs. $40 \%$ ) were not significantly different between the groups. This suggests that a key difference between this study and PROTECT may be in the usual care group rather than the NT-proBNP-guided treatment group. Patients enrolled in the usual care group of the this study had relatively frequent study-related clinic visits (median 10 visits over 15 months of follow-up) and adjustments to HF therapy (median of 4 adjustments), which represents a greater intensity of care (more akin to a disease management program) than would typically occur in routine clinical practice. Whether this frequency of clinical contact affected outcomes through mechanisms other than medication titration (e.g., by earlier detection and intervention on heart failure decompensation) is unknown. Although this study included both academic and community sites, the majority of this study sites had substantial focus and expertise in HF care which may have tended to lessen differences in the optimization of evidenced-based HF therapies between the study groups.

\section{Limitations}

This study has several important limitations. First, given the nature of the study intervention, the study was unblinded, which could be a potential source of bias. The design was based on an objective primary endpoint (cardiovascular death and heart failure hospitalization) that was adjudicated by a clinical events committee blinded to the treatment assignment in order to mitigate this bias. Second, although the study protocol discouraged measurement of NTproBNP in patients in the usual care group, some patients may have had NT-proBNP levels assessed at non-study sites or by non-study clinicians, which may have served to diminish the difference between study groups. Finally, patients in both groups had more frequent 
clinical encounters than would typically occur in clinical practice, which may have influenced the results.

\section{Conclusions}

In high-risk patients with HFrEF, a strategy of NT-proBNP-guided therapy was not more effective than a usual care strategy in improving outcomes.

\section{Supplementary Material}

Refer to Web version on PubMed Central for supplementary material.

\section{Acknowledgments}

We would like to acknowledge the contributions of the GUIDE-IT investigators, study coordinators, and the participating patients to the GUIDE-IT study. A listing of the GUIDE-IT investigators and study coordinators is shown in the Supplemental Material. The sponsor (the NHLBI) had no role in the design of the study but NHLBI staff did participate in study conduct, collection, management, analysis, and interpretation of the data; preparation, review, or approval of the manuscript; and decision to submit the manuscript for publication as members of the Steering Committee (Drs. Cooper, Leifer, and Desvigne-Nickens). Drs. Felker and Anstrom had full access to the all the data in the study and take responsibility for the integrity of the data and the accuracy of the data analysis.

\section{Sources of Funding}

The GUIDE-IT study was funded by National Institutes of Health grants HL105448, HL105451, and HL105457. Roche Diagnostics provided support for NT-proBNP testing. The views expressed in this article are those of the authors and do not necessarily represent the views of the National Heart, Lung, and Blood Institute; National Institutes of Health; or the United States Department of Health and Human Services.

\section{References}

1. Lee DS, Tu JV, Juurlink DN, et al. Risk-treatment mismatch in the pharmacotherapy of heart failure. JAMA. 2005; 294(10):1240-1247. [PubMed: 16160132]

2. Fonarow GC, Albert NM, Curtis AB, et al. Improving evidence-based care for heart failure in outpatient cardiology practices: primary results of the Registry to Improve the Use of EvidenceBased Heart Failure Therapies in the Outpatient Setting (IMPROVE HF). Circulation. 2010; 122(6): 585-596. [PubMed: 20660805]

3. Januzzi JL Jr, Sakhuja R, O'Donoghue M, et al. Utility of amino-terminal pro-brain natriuretic peptide testing for prediction of 1-year mortality in patients with dyspnea treated in the emergency department. Arch Intern Med. 2006; 166(3):315-320. [PubMed: 16476871]

4. Anand IS, Fisher LD, Chiang YT, et al. Changes in Brain Natriuretic Peptide and Norepinephrine Over Time and Mortality and Morbidity in the Valsartan Heart Failure Trial (Val-HeFT). Circulation. 2003; 107(9):1278-1283. [PubMed: 12628948]

5. Masson S, Latini R, Anand IS, et al. Prognostic Value of Changes in N-Terminal Pro-Brain Natriuretic Peptide in Val-HeFT (Valsartan Heart Failure Trial). J Am Coll Cardiol. 2008; 52(12): 997-1003. [PubMed: 18786480]

6. Eurlings LWM, van Pol PEJ, Kok WE, et al. Management of Chronic Heart Failure Guided by Individual N-Terminal Pro-B-Type Natriuretic Peptide Targets: Results of the PRIMA (Can PRobrain-natriuretic peptide guided therapy of chronic heart failure IMprove heart fAilure morbidity and mortality?) Study. Journal of the American College of Cardiology. 2010; 56(25):2090-2100. [PubMed: 21144969]

7. Troughton RW, Frampton CW, Yandle TG, Espiner EA, Nicholls MG, Richards AM. Treatment of heart failure guided by plasma aminoterminal brain natriuretic peptide (N-BNP) concentrations. Lancet. 2000; 355:1126-1130. [PubMed: 10791374] 
8. Januzzi JL Jr, Rehman SU, Mohammed AA, et al. Use of amino-terminal pro-B-type natriuretic peptide to guide outpatient therapy of patients with chronic left ventricular systolic dysfunction. J Am Coll Cardiol. 2011; 58(18):1881-1889. [PubMed: 22018299]

9. Pfisterer M, Buser P, Rickli H, et al. BNP-guided vs symptom-guided heart failure therapy: the Trial of Intensified vs Standard Medical Therapy in Elderly Patients With Congestive Heart Failure (TIME-CHF) randomized trial. Jama. 2009; 301(4):383-392. [PubMed: 19176440]

10. Berger R, Moertl D, Peter S, et al. N-Terminal Pro-B-Type Natriuretic Peptide-Guided, Intensive Patient Management in Addition to Multidisciplinary Care in Chronic Heart Failure: A 3-Arm, Prospective, Randomized Pilot Study. J Am Coll Cardiol. 2010; 55(7):645-653. [PubMed: 20170790]

11. Lainchbury JG, Troughton RW, Strangman KM, et al. N-Terminal Pro-B-Type Natriuretic PeptideGuided Treatment for Chronic Heart Failure: Results From the BATTLESCARRED (NT-proBNPAssisted Treatment To Lessen Serial Cardiac Readmissions and Death) Trial. J Am Coll Cardiol. 2009; 55(1):53-60. [PubMed: 20117364]

12. Felker GM, Hasselblad V, Hernandez AF, O'Connor CM. Biomarker-guided therapy in chronic heart failure: A meta-analysis of randomized controlled trials. American Heart Journal. 2009; 158(3):422-430. [PubMed: 19699866]

13. Troughton RW, Frampton CM, Brunner-La Rocca HP, et al. Effect of B-type natriuretic peptideguided treatment of chronic heart failure on total mortality and hospitalization: an individual patient meta-analysis. Eur Heart J. 2014; 35(23):1559-1567. [PubMed: 24603309]

14. Yancy CW, Jessup M, Bozkurt B, et al. 2013 ACCF/AHA Guideline for the Management of Heart Failure: A Report of the American College of Cardiology Foundation/American Heart Association Task Force on Practice Guidelines. Circulation. 2013; 128(16):e240-e327. [PubMed: 23741058]

15. Ponikowski P, Voors AA, Anker SD, et al. 2016 ESC Guidelines for the diagnosis and treatment of acute and chronic heart failure: The Task Force for the diagnosis and treatment of acute and chronic heart failure of the European Society of Cardiology (ESC)Developed with the special contribution of the Heart Failure Association (HFA) of the ESC. Eur Heart J. 2016

16. Felker GM, Ahmad T, Anstrom KJ, et al. Rationale and design of the GUIDE-IT study: Guiding Evidence Based Therapy Using Biomarker Intensified Treatment in Heart Failure. JACC Heart failure. 2014; 2(5):457-465. [PubMed: 25194287]

17. McMurray JJ, Packer M, Desai AS, et al. Angiotensin-neprilysin inhibition versus enalapril in heart failure. N Engl J Med. 2014; 371(11):993-1004. [PubMed: 25176015]

18. Bang H, Tsiatis A. Estimating medical costs with censored data. Biometrika. 2000; 87:329-343.

19. Andersen PK, Gill RD. Cox's Regression Model for Counting Processes: A Large Sample Study. Annals of Statistics. 1982; 10(4):1100-1120.

20. Felker GM, Whellan D, Kraus WE, et al. N-terminal pro-brain natriuretic peptide and exercise capacity in chronic heart failure: data from the Heart Failure and a Controlled Trial Investigating Outcomes of Exercise Training (HF-ACTION) study. American Heart Journal. 2009; 158(4 Suppl):S37-44. [PubMed: 19782787] 


\section{Key Points}

\section{Question}

Does a strategy of titrating therapy to a specific NT-proBNP target improve clinical outcomes in high-risk patients with heart failure and reduced ejection fraction?

\section{Findings}

In this randomized clinical trial including 894 adults, a strategy of NT-proBNP-guided therapy compared with usual care did not significantly improve time to first hospitalization or cardiovascular mortality (hazard ratio, 0.98).

\section{Meaning}

These findings do not support NT-proBNP-guided therapy for management of heart failure with reduced ejection fraction. 


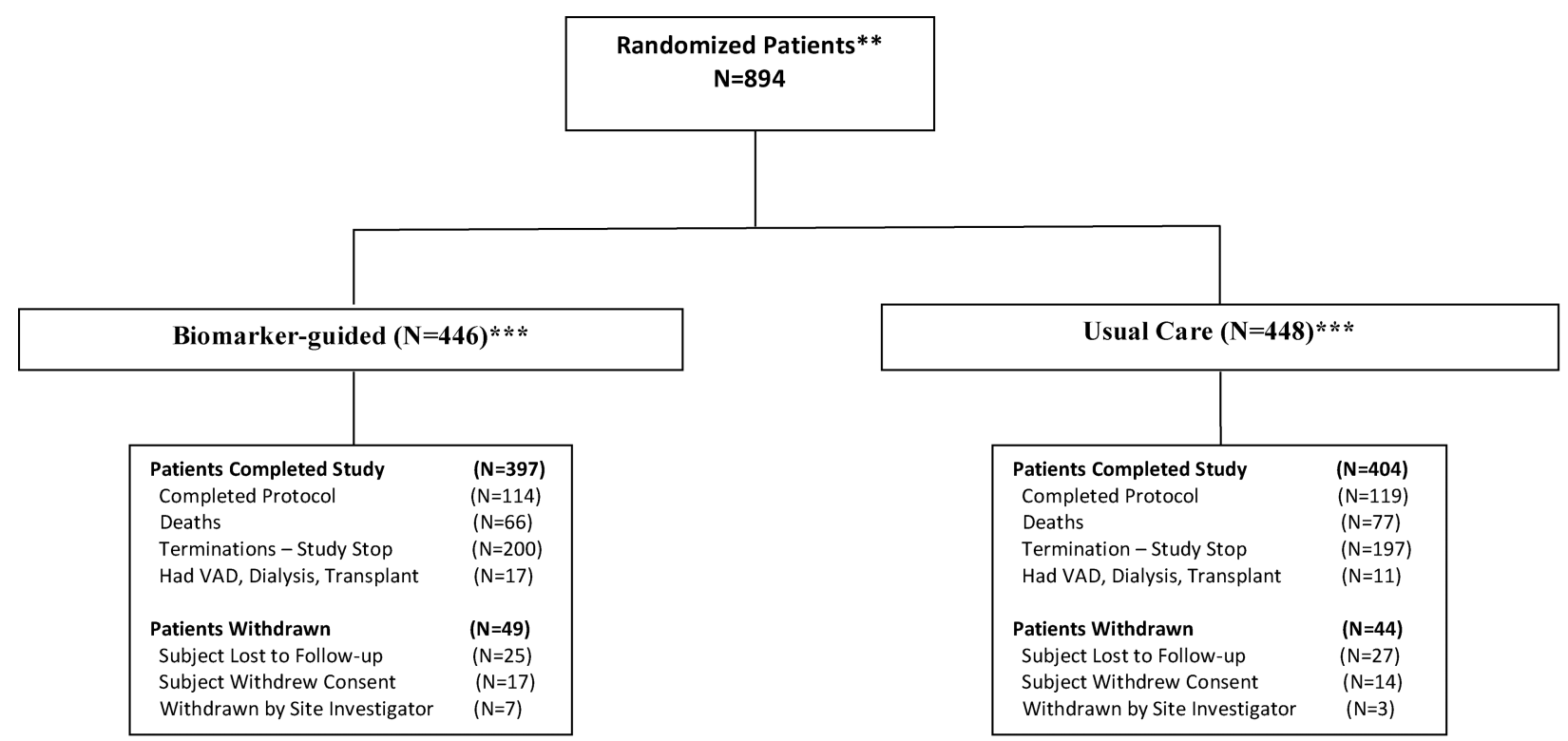

*Note: We did not collect the number of screened patients and the reasons for screen failures in this study because it is a multi-site study, so there are variations in the details of screening criteria by site.

**Note: All the randomized patients are included in the intent-to-treat population for the primary analysis. Patients who had study contact within 90 days prior to study stop are considered complete in this consort diagram.

*** In this Intent-to-Treat study, 446 patients in the Biomarker-guided arm and 448 in the Usual Care arm were included in the primary analysis.

Figure 1. Flowchart of Patient Accountability

This figure displays a flowchart of patient accountability, from the initial randomized patients through the number of patients who completed the study or withdrew from both the biomarker-guided and usual care groups. Data on the number of patients screened for eligibility are not available.

Abbreviations: VAD, ventricular assist device 


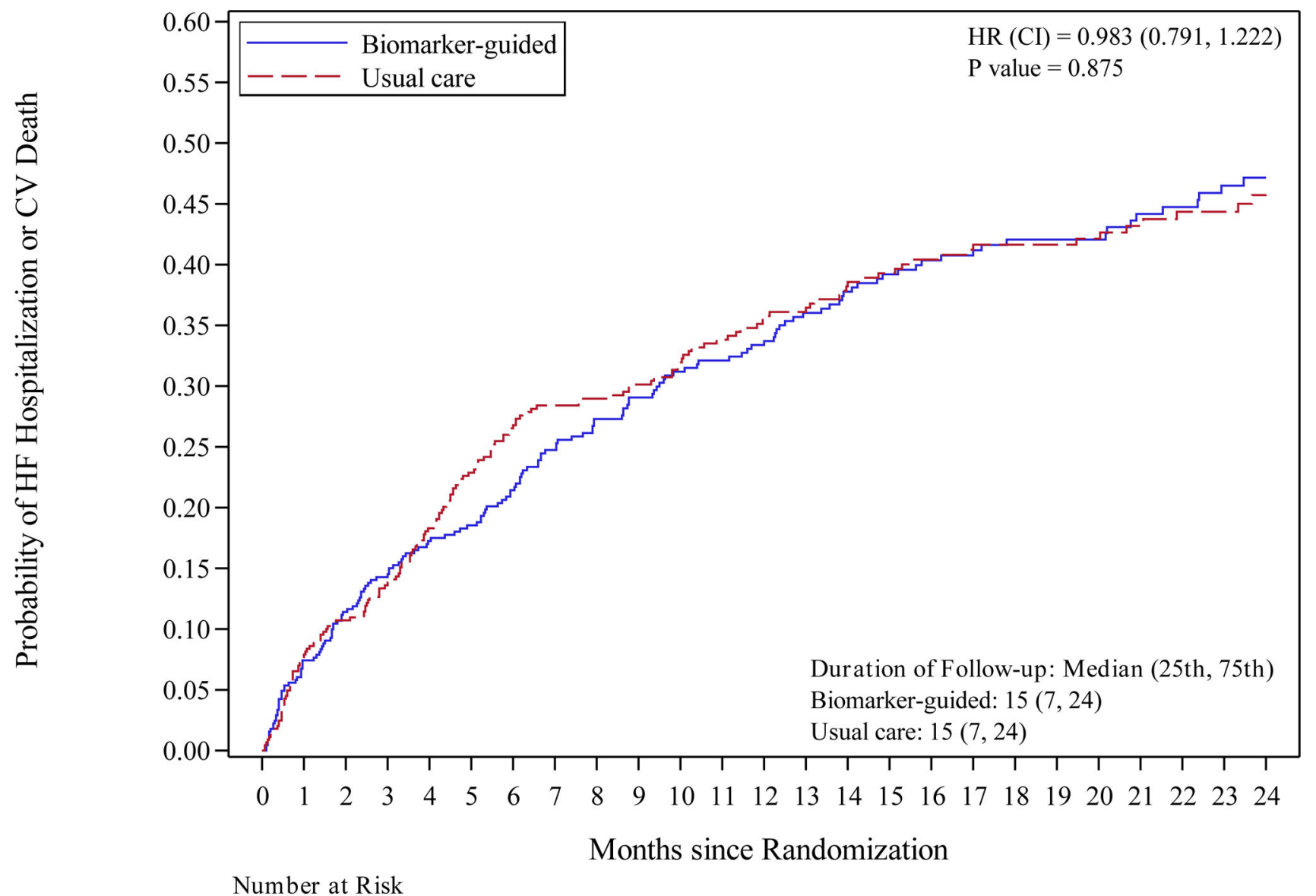

Biomarker-guided $44640537635533131529327225424222521820218517516615213512812812810093 \quad 85 \quad 78$ Usual care 448401381359330301278263257243227210199185175169153138138138115104909077 


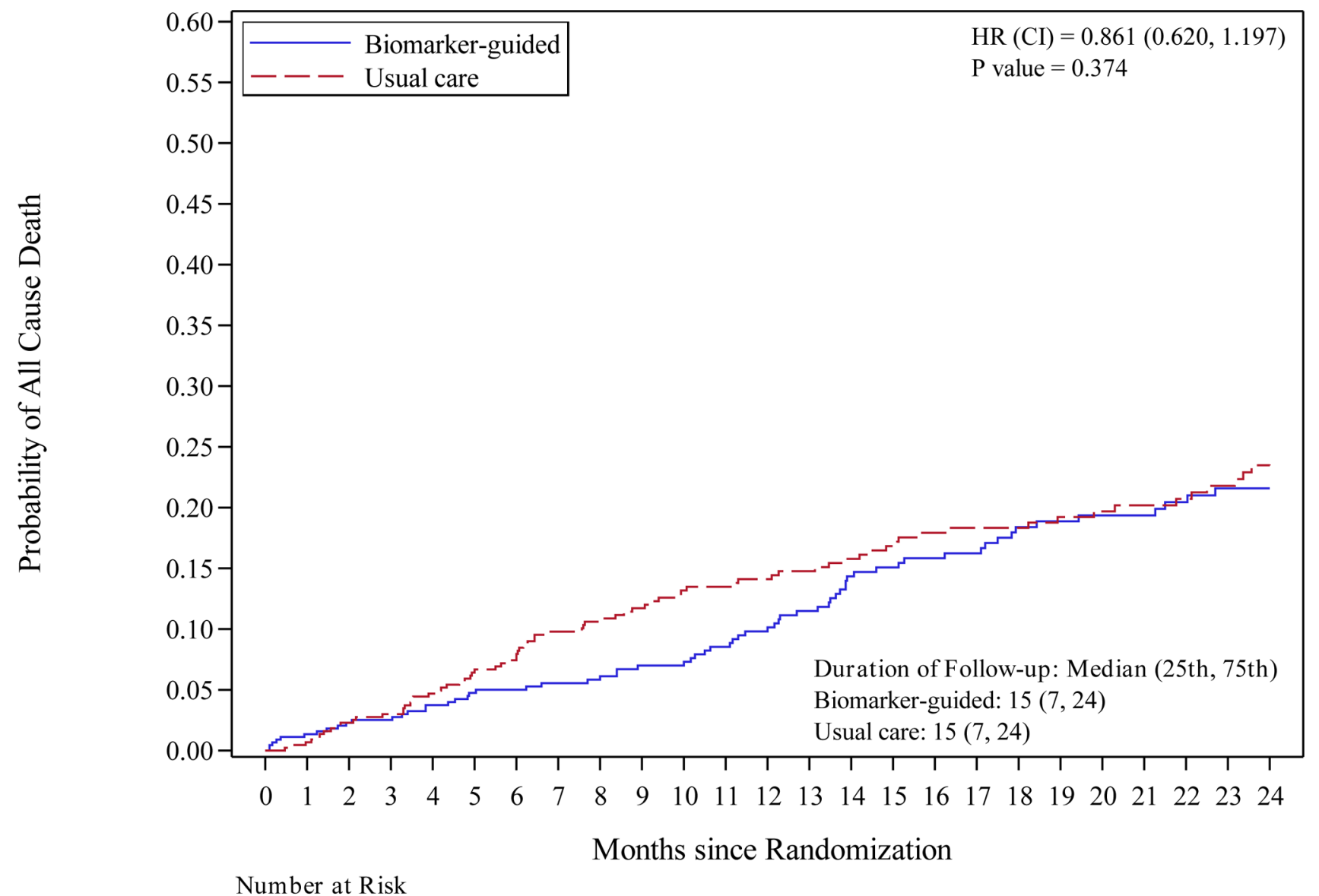

Biomarker-guided $\quad 446432418416391375371347327315304296275258239230221209186174167167145137137$

Usual care $\quad 448435423411391373358340323315297292273261248236217205205178171164150146134$

Figure 2. Primary Endpoint (Heart failure hospitalization or CV mortality) and All-Cause Mortality

Kaplan-Meier curves for: A) primary endpoint (heart failure hospitalization or CV

mortality); and B) all-cause mortality.

Abbreviations: CV, cardiovascular; $\mathrm{HF}$, heart failure 


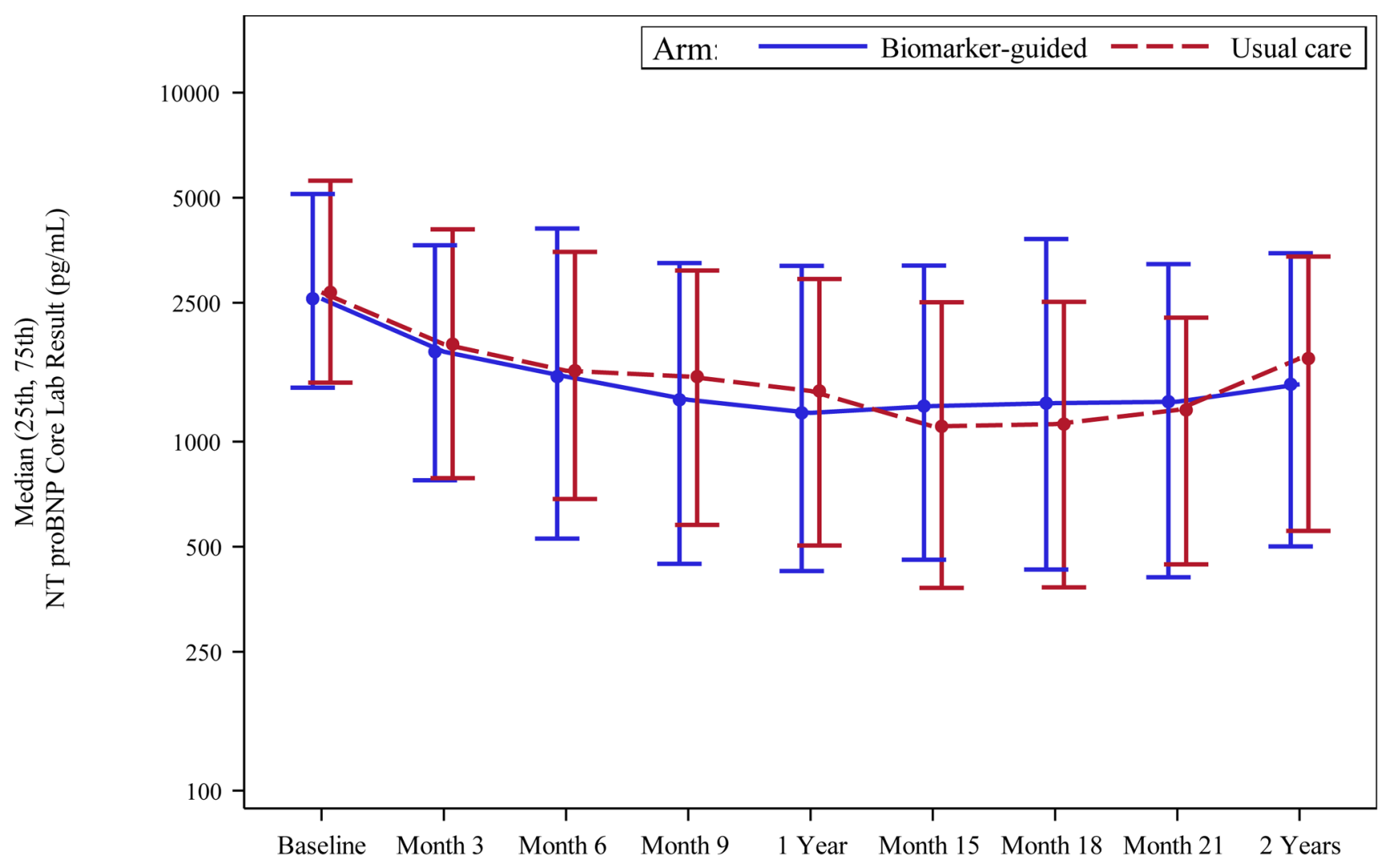

Biomarker-guided

426

355

305

263

228

180

153

123

105

Figure 3. Change in NT-proBNP Levels

NT-proBNP levels between the groups over time.

Abbreviations: NT-proBNP, amino-terminal pro-B-type natriuretic peptide. B-guided,

Biomarker guided therapy 


\section{Table 1}

\section{Baseline Characteristics}

\begin{tabular}{|c|c|c|}
\hline Characteristic & $\begin{array}{l}\text { NT-proBNP guided } \\
(\mathbf{n}=\mathbf{4 4 6})\end{array}$ & $\begin{array}{l}\text { Usual care } \\
(n=448)\end{array}$ \\
\hline Age (years) median $\left[25^{\text {th }}-75^{\text {th }}\right]$ & $62[51,70]$ & $64[54,72]$ \\
\hline Sex, No (\% female) & $139(31 \%)$ & $147(33 \%)$ \\
\hline \multicolumn{3}{|l|}{ Race, No (\%) } \\
\hline White & $230(54 \%)$ & $260(59 \%)$ \\
\hline Black & $168(39 \%)$ & $156(35 \%)$ \\
\hline Other & $35(7 \%)$ & $26(6 \%)$ \\
\hline Ethnicity, No. (\% Hispanic) & $30(7 \%)$ & $28(6 \%)$ \\
\hline Duration of HF (months) median $\left[25^{\text {th }}-75^{\text {th }}\right]$ & $12[1,65]$ & $16[1,61]$ \\
\hline Ejection fraction $(\%)$ median $\left[25^{\text {th }}-75^{\text {th }}\right]$ & $24[19,30]$ & $25[20,30]$ \\
\hline NYHA class at enrollment, No (\%) & $36(8 \%)$ & $23(5 \%)$ \\
\hline I & $218(50 \%)$ & $229(52 \%)$ \\
\hline II & $176(40 \%)$ & $182(41 \%)$ \\
\hline III & $8(2 \%)$ & $9(2 \%)$ \\
\hline \multicolumn{3}{|l|}{ IV } \\
\hline \multicolumn{3}{|l|}{ History of: No,(\%) } \\
\hline Ischemic heart disease & $203(46 \%)$ & $244(55 \%)$ \\
\hline Diabetes mellitus & $198(44 \%)$ & $212(47 \%)$ \\
\hline Atrial fibrillation & $162(36 \%)$ & $196(44 \%)$ \\
\hline Chronic kidney disease & $161(36 \%)$ & $169(38 \%)$ \\
\hline Systolic BP(mmHg) median $\left[25^{\text {th }}-75^{\text {th }}\right]$ & $114[102,128]$ & $114[101,128]$ \\
\hline Heart rate (beats/min) median $\left[25^{\text {th }}-75^{\text {th }}\right]$ & $77[68,87]$ & $76[67,86]$ \\
\hline NT-proBNP $(\mathrm{pg} / \mathrm{mL})$ median $\left[25^{\text {th }}-75^{\text {th }}\right]$ & $2632[1462-5235]$ & $2668[1481-5604]$ \\
\hline Creatinine $(\mathrm{mg} / \mathrm{dL})$ median $\left[25^{\text {th }}-75^{\text {th }}\right]$ & $1.3[1.1,1.7]$ & $1.3[1.1,1.7]$ \\
\hline Beta-blocker, No. $(\%)$ & $415(93 \%)$ & $416(93 \%)$ \\
\hline $\begin{array}{l}\text { Angiotensin Converting Enzyme inhibitor, Angiotensin receptor blocker, or Angiotensin receptor } \\
\text { blocker neprilysin inhibitor, No. }(\%)\end{array}$ & $345(77 \%)$ & $339(76 \%)$ \\
\hline Mineralocorticoid antagonist (\%) No. (\%) & $223(50 \%)$ & $217(48 \%)$ \\
\hline Implantable cardioverter defibrillator (\%) No (\%) & $182(41 \%)$ & $178(40 \%)$ \\
\hline Cardiac resynchronization therapy No (\%) & $87(20 \%)$ & $76(17 \%)$ \\
\hline
\end{tabular}


Table 3

Secondary Outcomes

\begin{tabular}{lcccc}
\hline & $\begin{array}{c}\text { NT-proBNP- } \\
\text { guided }\end{array}$ & Usual Care & Effect (95\% CI) & p-value \\
\hline Mortality, No. (\%) & $66(15 \%)$ & $77(17 \%)$ & $0.86(0.62-1.20)$ & 0.37 \\
CV mortality & $53(12 \%)$ & $57(13 \%)$ & $0.94(0.65-1.37)$ & 0.75 \\
Non-CV mortality & $13(3 \%)$ & $20(5 \%)$ & $0.66(0.33-1.32)$ & 0.24 \\
First HF hospitalization, No (\%) & $147(33 \%)$ & $141(32 \%)$ & $1.04(0.82-1.31)$ & 0.76 \\
Total HF hospitalizations (No.) & 350 & 277 & $1.29(0.97-1.72)$ & $0.083^{*}$ \\
Days alive and not hospitalized for CV reasons, mean (SD) & 581 days (14.4) & 562 days (15.1) & $19.26(-21.58-60.10)$ & $0.36^{\#}$ \\
\hline
\end{tabular}

Abbreviations: CI, confidence interval; CV, cardiovascular;

All other abbreviations can be found in Table 1.

* Based on Andersen-Gill Intensity model.

\# Based on Bang-Tsiatis portioned estimator 